\title{
Correction to: End-of-life geostationary satellite removal using realistic flat solar sails
}

\author{
Hao Mei ${ }^{1} \cdot$ Christopher J. Damaren ${ }^{1} \cdot$ Xingqun Zhan $^{2}$
}

Published online: 14 June 2021

(c) Shanghai Jiao Tong University 2021

\section{Correction to:}

\section{Aerospace Systems}

https://doi.org/10.1007/s42401-021-00089-8

Due to an unfortunate oversight the Eq. (8), Tables 2 and 4 has been given erroneously. It should read (Tables 2 and 4)

$$
\underbrace{\frac{d}{d t}\left(\begin{array}{c}
a \\
e \\
i \\
\omega \\
\Omega \\
\theta
\end{array}\right)}_{\dot{\boldsymbol{x}}}=\underbrace{\left(\begin{array}{ccc}
\frac{2 a^{2}}{\sqrt{\mu a\left(1-e^{2}\right)}} e \sin (\theta) & \frac{2 a^{2}}{\sqrt{\mu a\left(1-e^{2}\right)}}(1+e \cos (\theta)) & 0 \\
\sqrt{\frac{a\left(1-e^{2}\right)}{\mu}} \sin (\theta) & \sqrt{\frac{a\left(1-e^{2}\right)}{\mu} \frac{2 \cos (\theta)+e\left(1+\cos ^{2}(\theta)\right)}{1+e \cos (\theta)}} & 0 \\
0 & 0 & \sqrt{\frac{a\left(1-e^{2}\right)}{\mu}} \frac{\cos (\omega+\theta)}{1+e \cos (\theta)} \\
-\sqrt{\frac{a\left(1-e^{2}\right)}{\mu}} \frac{\cos (\theta)}{e} & \sqrt{\frac{a\left(1-e^{2}\right)}{\mu}} \frac{(2+e \cos (\theta)) \sin (\theta)}{e(1+e \cos (\theta))} & -\sqrt{\frac{a\left(1-e^{2}\right)}{\mu}} \frac{\sin (\omega+\theta)}{\tan (i)(1+e \cos (\theta))} \\
0 & 0 & \sqrt{\frac{a\left(1-e^{2}\right)}{\mu}} \frac{\sin (\omega+\theta)}{\sin (i)(1+e \cos (\theta))} \\
\sqrt{\frac{a\left(1-e^{2}\right)}{\mu}} \frac{\cos (\theta)}{e} & -\sqrt{\frac{a\left(1-e^{2}\right)}{\mu}} \frac{(2+e \cos (\theta)) \sin (\theta)}{e(1+e \cos (\theta))} & 0
\end{array}\right)}_{\text {denote as } \mathbf{P}(\mathbf{x})} \underbrace{\left(\begin{array}{c}
f_{r} \\
f_{\theta} \\
f_{z}
\end{array}\right)+\underbrace{0}}_{\text {denote as } \mathbf{b}(\mathbf{x})}
$$

The original article can be found online at https://doi.org/10.1007/ s42401-021-00089-8.

Hao Mei

hao.mei@mail.utoronto.ca

Christopher J. Damaren

damaren@utias.utoronto.ca

Xingqun Zhan

xqzhan@sjtu.edu.cn

1 University of Toronto Institute for Aerospace Studies, 4925 Dufferin Street, Toronto, ON M3H 5T6, Canada

2 Shanghai Jiaotong University School of Aeronautics and Astronautics, 800 Dongchuan Road, Shanghai 200240, China 
Table 2 The GEO graveyard region

\begin{tabular}{ll}
\hline Property & Requirement \\
\hline Perigee altitude & A minimum increase of $235 \mathrm{~km}+\left(100 \cdot \mathrm{C}_{\mathrm{R}} \cdot A / m\right)$ \\
& $235 \mathrm{~km}:$ the sum of the upper altitude of the GEO \\
& protected region $(200 \mathrm{~km})$ and the maximum \\
descent due to luni-solar and geo-potential & perturbations $(35 \mathrm{~km})$ \\
& $\mathrm{C}_{\mathrm{R}}:$ the solar radiation pressure $(\mathrm{SRP})$ coefficient \\
& $A / m:$ the area to dry mass ratio \\
Eccentricity & {$[0,0.003]$}
\end{tabular}

Table 4 Comparisons between the ideal solar sails and the realistic flat solar sails

Solar sail thrust model

\begin{tabular}{ll} 
Ideal sail & $\boldsymbol{f}_{\text {ideal }}=\left[2 P_{\odot} \cdot(A / m) \cdot \cos ^{2} \alpha\right] \boldsymbol{n}$ \\
Realistic flat Sail & $\boldsymbol{f}_{\text {real }}=\boldsymbol{f}_{n}+\boldsymbol{f}_{t}$ \\
& $\boldsymbol{f}_{n}=\left(P_{\odot} \frac{A}{m}\left[(1+\tilde{r} s) \cos ^{2} \alpha+B_{f}(1-s) \tilde{r} \cos \alpha+(1-\tilde{r}) \frac{\xi_{f} B_{f}-\xi_{b} B_{b}}{\xi_{f}+\xi_{b}} \cos \alpha\right]\right) \boldsymbol{n}$ \\
& $\boldsymbol{f}_{t}=\left(P_{\odot} \frac{A}{m}(1-\tilde{r} s) \cos \alpha \sin \alpha\right) \boldsymbol{t}$ \\
\hline
\end{tabular}

Control angle constraints

Ideal sail

Realistic flat sail

System dynamics

Ideal sail

Realistic flat sail $\alpha \in\left[0^{\circ}, 90^{\circ}\right], \delta \in\left[0^{\circ}, 360^{\circ}\right]$

$\alpha \in\left[0^{\circ}, 85^{\circ}\right], \delta \in\left[0^{\circ}, 360^{\circ}\right]$

The original article has been corrected. 